\title{
Chinese translation and psychometric testing of the Humour Styles Questionnaire Children Version (C- HSQC) among Hong Kong Chinese primary-school students
}

\author{
Kelly Ku
}

Hong Kong Baptist University

kellyku@hkbu.edu.hk

\section{Sammy Ho}

Hong Kong Baptist University sammyho@hkbu.edu.hk

\section{Edmund Chan}

Hong Kong Baptist University 11467479@life.hkbu.edu.hk

\section{Maria Chik}

Hong Kong Baptist University pychik@hkbu.edu.hk

\section{Dennis Chan}

Hong Kong Baptist University dennis@hkbu.edu.hk

\begin{abstract}
In the current study, the Humour Styles Questionnaire Children Version (HSQC) was translated into Chinese. The psychometric properties of the Chinese version of the Humour Styles Questionnaire Children version $(C-H S Q C)$ were examined. The C-HSQC and psychological measures on humour, self-esteem, depression, and coping behaviors were administered to a sample of 361 primary-five and -six students in Hong Kong. Confirmatory factor analyses, concurrent, convergent, and discriminant validity were assessed to examine the construct validity of the questionnaire. The results indicated a moderately strong fit of the model of the hypothesized four-factor structure of two adaptive (i.e., affiliative and selfenhancing) and two maladaptive (i.e., aggressive and self-defeating) humour styles. Results
\end{abstract}


provided good support for the use of the C-HSQC in measuring humour of Hong Kong children.

Keywords: humour, psychometric evaluation, children.

\section{Background of research}

\subsection{Concept of humour}

Humour is recognised as a human character trait and a prevalent and pervasive human practice (Tapley 2006). Humour research has been challenged by lacking a consensual and universal definition (Ruch 1998). Humour may carry different meanings subject to its context. As highlighted by Martin (1998: 15-16), humour comprises a wide range of concepts including but not limited to amusement, wit, ridicule, comedy, whimsy, and satire. Given the multifaceted nature of humour, any single broad theory is inadequate in capturing all of its qualities (Foot 1986). Ruch (1998: 12-13) and Martin (1998: 6-7) considered that the term "humour" per se neutral with no prior positive or negative evaluative assumptions. Yet generally contemporary researchers conceptualise humour as a multidimensional construct incorporating both positive (e.g., affiliative and self-enhancing) and negative (e.g., aggressive and self-defeating) dimensions because their differential effects on psychological outcomes are evidenced (e.g., Kuiper \& McHale 2009; Martin et al. 2003; see below discussion).

\subsection{The adult and adolescent humour styles questionnaire and related empirical findings}

Martin and his colleagues (2003) developed the Humour Styles Questionnaire (HSQ) for adults to examine specifically the adaptive and the maladaptive aspects of humour, as other current self-reported humour measures (e.g., Situational Humour Response Questionnaire (SHRQ; Martin \& Lefcourt 1984), Sense of Humour Scale (SHS; McGhee 1996), Revised Sense of Humour Questionnaire (SHQ-6; Svebak 1996), and Multidimensional Sense of Humour Scale (MSHS; Thorson \& Powell 1993)) do not make such distinction explicitly. The HSQ questionnaire assesses two adaptive (i.e., affiliative and self-enhancing) and two maladaptive (i.e., aggressive and self-defeating) humour styles. Affiliative humour assesses the extent to which individuals are able to amuse others with jokes, funny stories, and other forms of benevolent humour. Used in this way, humour enhances interpersonal relationships and reduces interpersonal tensions. Self-enhancing humour refers to the tendency to maintain a humorous perspective on life, employ humour in coping with stress, and cheer oneself up for mastering difficult or stressful situations. On the other hand, aggressive humour describes the tendency to the use of teasing, ridicule, sarcasm, and disparagement to enhance oneself but put down others. Self-defeating humour defines the tendency to use self-disparaging and ingratiating humour in an excessive attempt to gain the approval of others in order to facilitate one's interpersonal and social relationships.

The HSQ reported a satisfactory factor structure $(\mathrm{GFI}=.91, \mathrm{AGFI}=.90$ and RMSEA $=$ .048) (Martin et al. 2003: 57) and test-retest reliability, supporting its construct validity and internal consistency (Martin et al. 2003: 59). It was found that individuals who use humour to facilitate interpersonal relationships are likely to engage in self-enhancing uses of humour. Whereas individuals who engage in disparagement humour are more likely to amuse others by ingratiating themselves so as to gain approval from their in-group members. In addition, those who frequently tell jokes and amuse others to enhance relationships also show some tendency 
to engage in put-down or disparagement humour. The males showed a slightly stronger tendency to do so as compared to the females (Martin et al. 2003: 60). These findings imply that some elements of aggression (e.g., teasing a friend) might be socially-oriented and are therefore being incorporated into affiliative humour for relationship building.

Numerous studies have found empirical support for the HSQ and the differential relationships between adaptive and maladaptive humour styles with various aspects of psychological well-being among adults. For example, individuals who engaged in affiliative and self-enhancing humour reported higher self-esteem (Kuiper \& McHale 2009), whereas fewer indications of depressive symptoms (Cheung \& Yue 2012: 359; Taher et al. 2008: 559). While self-defeating humour was found to be associated with higher level of depression (Taher et al. 2008: 559) and lower level of self-esteem (Kuiper \& McHale 2009: 370). Aggressive humour, on the other hand, was related to neither level of depression (Dozois et al. 2009: 591592) nor self-esteem (Martin et al. 2003: 66). As compared to other measures of humour, the HSQ allows more in-depth investigation and statistical relationships of humour and other psychological constructs to be established, given its four relatively distinct dimensions. The Humour Styles Questionnaire (HSQ) has been extended to the use of adolescents. The adolescent version demonstrated an acceptable internal consistency and adequate validity established by Erickson and Feldstein (2007: 263-265). The reliability coefficients for the four dimensions of humour ranged from .58 to .81 , with relatively lower coefficients reported for the maladaptive humour styles. Inter-correlations among the four dimensions were comparable to those reported by Martin et al. (2003: 59-60). For the adolescent sample, the dimension of adaptive humour were found to be positively related to personal adjustment (i.e., a measure of adaptive functioning) but negatively associated with depression. This finding was in line with Freiheit et al.'s (1998: 41-42) study revealing a negative relationship between coping humour and depression among adolescents. Self-enhancing humour and self-defeating humour was found to promote approach and avoidance coping respectively. These results indicated that positive humour might be used as an effective form of active coping strategy. It must be noted that the affiliative and aggressive humour styles were not related, suggesting that adolescents tend not to use humour to enhance relationships at the expense of putting others down. One underlying reason might be that gender difference was not examined in Erickson and Feldstein's study (2007), as a previous study of early adolescents (Führ 2002: 296) has shown that boys tend to use more aggressive and sexual strategies in coping humour as compared to girls.

\subsection{The need for humour measurement in children}

While the literature on humour research related to children is burgeoning, empirical studies have been measuring children's humour with items borrowed from adult humour scales. The Multidimensional Sense of Humour Scale designed for children has been developed by Dowling and Fain (1999), revised by Dowling et al. (2003), and validated by Ho et al. (2012) in a Chinese context. However, the scale mainly captured the adaptive functions of humour for children. Aside the raising interest in children's use of humour, researchers' understanding of it, in particular the maladaptive functions of humour in children, has been restrained by the availability of measurement.

In view of the above, Fox et al. (2013) modified the HSQ to create a children version (i.e., HSQC) suitable for the age of nine to 15. For the usage of HSQ for children, a number of procedures were conducted to enhance clarity in comprehension and response. Ambiguous, difficult, and negatively-worded items were rephrased, retaining just four negatively worded items with the total number of items reduced from 32 to 24 . Response options were changed from a seven-point to a four-point Likert scale of attitude agreement (i.e., strongly disagree to 
strongly agree). With the above procedures to refine the instrument for the use of children, a strong four-factor structure for both primary- and secondary-age children was established, with a stronger model fit for sample of secondary students as compared to primary five and six children. The Cronbach alphas coefficients for the four dimensions ranged from .63 to .75, with relatively lower coefficients reported for self-defeating humour. Test-retest reliabilities for the four humour dimensions varied between .66 and .81. As expected, affiliative and self-defeating humour were positively and negatively linked to global self-worth. This is in line with the findings of Chik (2001) and Søbstad and Lillemyr (2010) which have found that a more positive view on one's humoristic self was related to higher self-esteem. Besides, affiliative humour was negatively associated with depression for boys but not girls. Aggressive humour was positively correlated with depression and negatively related to global self-worth for girls only, supporting the view that the use of aggressive humour in girls is more strongly associated with depression and low self-esteem compared to boys. Different from the results of previous studies in adults and adolescents (Erickson \& Feldstein 2007; Martin et al. 2003), self-enhancing humour was not related to either depression or global self-worth for both boys and girls. These results, however, were generally consistent with previous study (Sugimura et al. 2014: 571) showing that using humour to cope was not related to depressive symptoms among a group of primary two children.

\section{The present study}

In view of the need in contemporary humour research measurement that goes beyond capturing only the adaptive dimensions of humour, and that is suitable for the use of children, the HSQ Children version (HSQC) has demonstrated itself as a promising instrument. Currently the HSQC has only been tested in very limited samples in western studies. More empirical evidence is needed for testing its psychometric properties for its applicability in different cultures.

The present study has two aims. We translated HSQC into Chinese and examined the psychometric properties (i.e., internal consistency and validity) for its use among Hong Kong primary-school children. It is believed that the present study will contribute to the scant literature on humour measurement available to children, understanding the adaptive and maladaptive aspects of humour in children, as well as serving as a foundation for studying perseverance and adversity in children.

\section{Methodology}

\subsection{Participants}

A convenience sample of 361 Chinese primary five and six students (198 boys and 160 girls; three of them did not indicate their gender) was drawn from two primary schools in Hong Kong. Participation was voluntary with informed consent from the school principals, parents, and students themselves. The mean age of participants was $11.75(\mathrm{SD}=.71)$.

\subsection{Procedures}

A paper-and-pencil questionnaire was distributed during a regular class period under the supervision of a class teacher and a research assistant. The research assistant gave instructions to the students and answered questions from students about uncertainty during the administration of the questionnaire. Students were assured that information provided would be 
kept anonymous and confidential. The participants spent an average of 15 minutes to complete the questionnaire.

\subsection{Measures}

The questionnaire contained 76 questions of five instruments as described below (in English back-translation).

\subsubsection{The Chinese version of the Humour Styles Questionnaire for Children (C-HSQC)}

The of HSQC (Fox et al., 2013) is a self-reported measure of 24-item and four subscales measuring two adaptive (affiliative, e.g., "I find it easy to make other people laugh", $\alpha=.87$; self-enhancing, e.g., "If I am feeling sad, I can cheer myself up by thinking of funny things", $\alpha$ $=.75$ ) and two maladaptive humour dimensions (aggressive, e.g., "If someone makes a mistake, I will often tease them about it", $\alpha=.74$; self-defeating, e.g., "I let people laugh at me and make fun of me more than I should", $\alpha=.70$ ). The reported Cronbach's alpha was above .70 for the questionnaire. Children were required to rate each item on a four-point Likert scale (one $=$ totally disagree to four $=$ totally agree). The higher score implies a more frequent use of the humour style.

Following the translation guidelines and procedures of Hambleton (2005), the original 24item HSQC was translated into Chinese language using the translate-back-translate method. We employed three independent translators with a degree or master degree in language or translation. The questionnaire was then back-translated by another three independent translators with similar qualifications. We ensured inter-rater reliability by having three independent raters assessing on the phonetic and semantic similarity between the original and the back-translated version. Discrepancy was sorted out by the researchers and the translators, with revisions sent for independent similarity rating until the overall percent agreement reached above $85 \%$. The researchers then made a final round of fine-tuning to ensure compatibility to original questionnaire and proper comprehension level for children.

The translated C-HSQC was pilot-tested with a class of primary six students $(N=34)$ to ascertain the readability and understanding. Based on the feedback from students, minor modifications were subsequently made by the researchers.

\subsubsection{The Chinese version of the Multidimensional Sense of Humour Scale for Children (C- MSHSC)}

The C-MSHSC (Ho et al. 2012) contains 17 items measuring three different dimensions of humour: Humour Creation (e.g., "I can make other people laugh"; six items; $\alpha=.91$ ), Coping with Humour (e.g., "Jokes and funny stories help me get through tough times"; five items; $\alpha=$ .90), and Humour Appreciation (e.g., "I like to hear a funny story"; six items; $\alpha=.87$ ). Children were asked to rate each item based on a five-point Likert-type scale, ranging from never (one) to always (five). The overall C-MSHSC score indicates level of sense of humour.

\subsubsection{Rosenberg's Self-esteem Scale (RSE)}

The Chinese version of the 10-item RSE (Rosenberg 1965) was used to assess the selfacceptance aspect of self-esteem of children. The items are presented on a four-point Likert scale from one (strongly disagree) to four (strongly agree) (Chik 2001). Total score ranges from 10 to 40, with higher score indicates higher level of self-esteem. Sample item includes "I am able to do things as well as most other people". We deleted one reversed scoring items "I wish I could have more respect for myself" due to its negative item-total correlation $(r=-.35$; the 
item-total correlations for the remaining nine items varied between .43 to .67), retaining nine items. The alpha coefficient of the current sample of the nine-item version was .87 .

\subsubsection{The Chinese version of the Centre for Epidemiological Studies Depression Scale for Children (CES-DC)}

The Chinese version of the CES-DC is a 20-item self-report questionnaire measuring the severity of depressive symptoms among children (Li et al. 2010a). Participants are required to indicate how much they have felt (zero $=$ "Not at all" to three $=$ "A lot") to individual statements reflecting depressive experiences during the past week. Possible scores range from zero to 60, with higher scores indicating more severe depressive symptoms. Scores above a cutoff score of 15 indicates significant levels of depression. The Cronbach's alpha reported was .89.

\subsubsection{Coping Behaviour Checklist for Chinese Children (CBC-CC)}

The five-item CBC-CC (Li et al., 2010b) assesses five different coping strategies in dealing with stressful events, they are social support (e.g., talking to parents, friends, or school teachers), problem solving (e.g., employing coping strategies), escape-avoidance (e.g., resort to TV watching, play video games), distancing (e.g., attempt to forget the entire experience), and self-control (e.g., staying calm). Children rate how often they would adopt each strategy (zero = "Hardly ever", one = "Sometimes" and three = "Often") when faced with a stressful event. Higher scores represent a higher frequency of occurrence of the behaviour described by the item. The reported internal consistency was .71 and the test-retest reliability was .83 .

\subsection{Statistical analyses}

Data cleansing was first performed. A total of 341 cases were retained. 20 cases were discarded due to problematic responding patterns, i.e., Christmas-Tree pattern. Christmas-Tree pattern is a "pattern of position" (Freire \& Tosch, 2015: 5), which describes respondents' choosing the options of a questionnaire in a pattern of artistic way, like drawing a Christmas tree. This pattern of responding occurs when respondents want to complete the survey quickly and conveniently without actually processing the questions (Zapin 2013). Missing data pattern was assessed by the Little's Missing Completely at Random (MCAR) Test. It revealed that the data was not missing completely at random $\left(\chi^{2}=508.979, d f=453, p=0.035\right)$. In view of this, it is not suitable for imputation of the missing data by using Expectation Maximisation (EM). Given that missing data would hinder subsequent factor analysis (Huck 2012), and that less than 15\% of missing data was acceptable for deletion (Hair et al. 2010), it was identified that 34 missing cases were suitable for removal. We retained a total of 307 cases for further analysis.

\section{Results}

\subsection{Construct validity of the Chinese version of the Humour Styles Questionnaire for Children (C-HSQC)}

Confirmatory factor analysis (CFA) using maximum likelihood estimation (SPSS AMOS 22.0) was conducted to examine the replicability of the original four-factor solution of the HSQ established by Fox et al. (2013: 311). The four-factor as latent variables were allowed to covary (the correlations among the four factors ranged from -.05 to .47). The parameter estimates of this four-factor model are shown in Figure 1. 


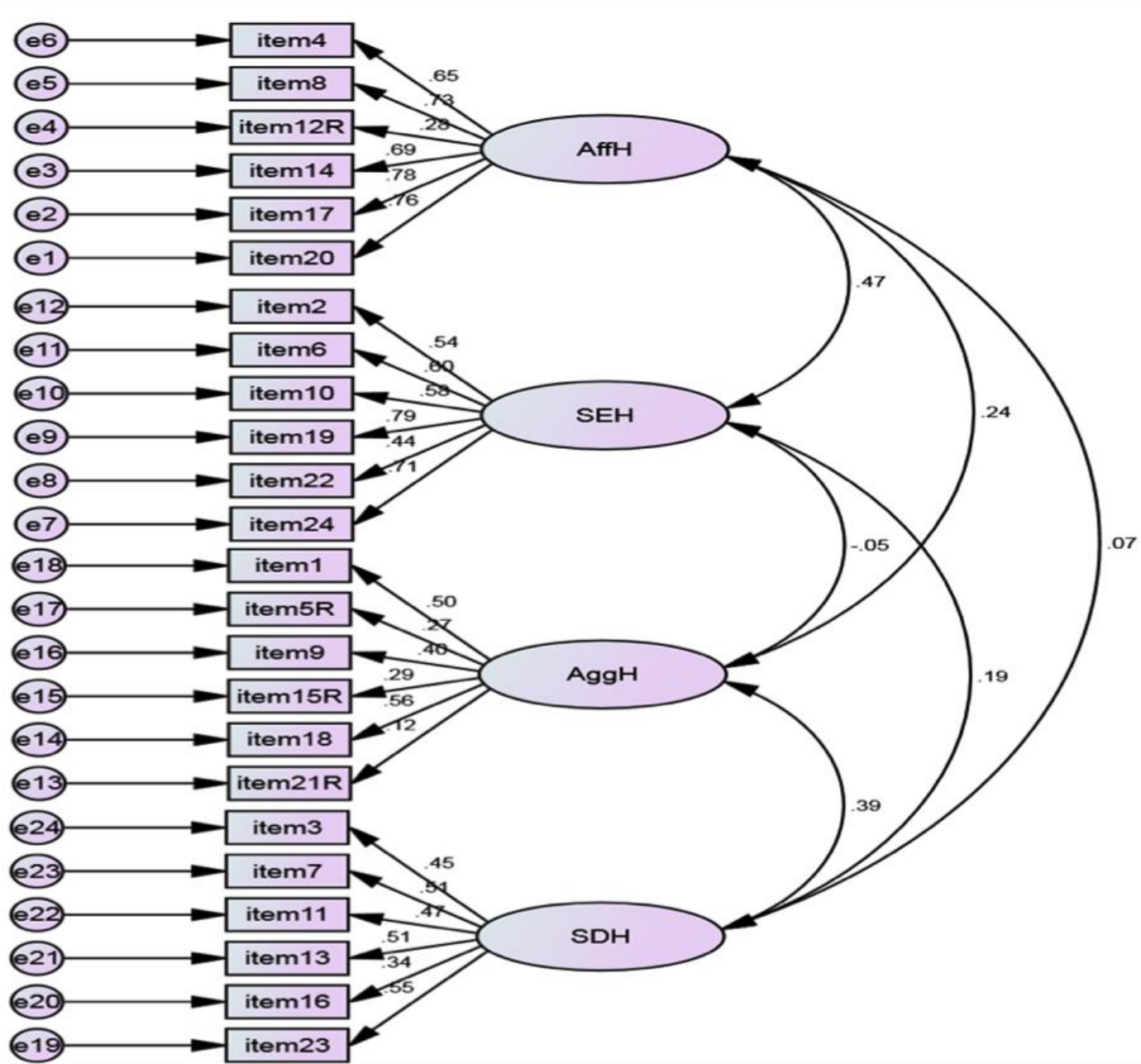

Figure 1. Confirmatory factor analysis for the four-factor model of the Chinese Humour Styles Questionnaire (C-HSQC) for Children. Note: AffH: Affiliative Humour; SEH: Selfenhancing Humour; AggH: Aggressive Humour; SDH: Self-defeating Humour.

Results suggested that a moderate fit to the data $(\mathrm{CMIN} / \mathrm{DF}=2.12, \mathrm{CFI}=.83, \mathrm{GFI}=.87$, TLI= .81 , and RMSEA $=.06(90 \% C I=.05-.07))$. Internal consistency coefficients of the four dimensions affiliative, self-enhancing, aggressive, and self-defeating humour were .81, .78, .48 , and .63 respectively. This result was in line with previous studies (Erickson \& Feldstein 2007: 263-264; Fox et al. 2013: 309-310), showing that the adaptive humour dimensions were more consistent as compared to the maladaptive humour dimensions.

The standardised regression coefficients (ranged from .12 to .79) and the corrected itemtotal correlations of the four humour dimensions (ranged from -.08 to .54) suggested that the values of four reversed-scoring items (items five, 12, 15, and 21) were particularly low, suggesting that these items should be modified in the Chinese context in order to improve the model fit and internal consistency of the scale.

To ensure the above four-factor model of C-HSQC is sufficiently equivalent for boys and girls, tests of configural invariance and construct-level metric invariance were performed (Kline 2011: 252-253; Millsap \& Olivera-Aguilar 2012: 382). Configural invariance is assessed by obtaining model fit without equality constraints of parameters imposed across two groups. Metric invariance is examined after evidencing configural invariance. Same analysis was conducted with cross-group equality constraints imposed on factor loadings. The chisquare difference based on the configural and metric models was then calculated. Statistically insignificant result indicated measurement invariance. Although various procedures to test for 
invariance are available (e.g., Byrne 2010; Chen et al. 2005), the present study followed those of Gaskin (2012) and the model fit statistics were shown in Table 1. The configural invariance test showed adequate fit statistics $\left(\chi^{2} / \mathrm{df}=1.56, \mathrm{CFI}=.83, \mathrm{GFI}=.87, \mathrm{TLI}=.81\right.$ and $\mathrm{RMSEA}=$ $.06(90 \%$ CI of RMSEA $=.04-.05))$, implying that the factor structure was equal to both boys and girl. The metric invariance test revealed that the resulting $\chi^{2}$ difference value was significant $\left(\chi^{2}(24)=46.716, p<.01\right)$,suggesting the factor structure was not the same for both boys and girls. According to Gaskin (2012), Items with tremendously different standardized regression weight from both groups should be identified and removed. Consequently, three problematic items on the factor Aggressive Humour were discovered and removed. Item one had a loading of -.06 for boys and .64 for girls. Item nine had a loading of - .55 for boys and .31 for girls. Item 18 had a loading of -.39 for boys and .47 for girls. Negative factor loading indicated that these items were negatively associated with the factor (Sheskin 2011: 1635) and this was usually caused by negatively-worded item (Valente 2002: 158). Given the negative loadings only appeared for boys, it is inferred that boys and girls interpreted these items underlying the dimension aggressive humour differently.

Table 1. Model fit statistics across gender samples

\begin{tabular}{|c|c|c|c|c|c|c|c|}
\hline Model & $\chi^{2}$ & df & $\chi^{2 / d f}$ & CFI & GFI & TLI & RMSEA \\
\hline Good model fit & & & $\leq 2.00$ & $\geq 0.90$ & $\geq 0.90$ & $\geq 0.95$ & $\leq 0.06$ \\
\hline $\begin{array}{l}\text { Configural } \\
\text { Samples)* }\end{array}$ & 521.62 & 246 & 2.12 & 0.83 & 0.87 & 0.81 & 0.06 \\
\hline Configural (24 items) & 765.16 & 492 & 1.56 & 0.83 & 0.83 & 0.81 & 0.04 \\
\hline Metric (24 items) & 811.88 & 516 & 1.57 & 0.82 & 0.82 & 0.81 & 0.04 \\
\hline Configural (21 items) & 577.69 & 366 & 1.58 & 0.86 & 0.85 & 0.84 & 0.04 \\
\hline Metric (21 items) & 597.83 & 387 & 1.55 & 0.86 & 0.85 & 0.85 & 0.04 \\
\hline $\begin{array}{l}\text { Configural (Combined } \\
\text { Samples, } 21 \text { items)** }\end{array}$ & 363.16 & 183 & 1.98 & 0.88 & 0.90 & 0.86 & 0.06 \\
\hline
\end{tabular}

Note: *Configural (Combined Samples) indicated the original model (24 items) based on combined samples $(N=307)$. **Configural (Combined Samples, 21 items) indicated the modified model (21 items) based on combined samples $(N=307)$.

A second round of invariance analysis was conducted after the removal of items one, nine and 18 , leaving the scale with 21 items. The configural invariance test on 21 items passed with adequate model fit $\left(\chi^{2} / \mathrm{df}=1.58, \mathrm{CFI}=.86, \mathrm{GFI}=.85, \mathrm{TLI}=.84\right.$ and $\mathrm{RMSEA}=.04(90 \% \mathrm{CI}$ of RMSEA $=.04-.05))$. The metric invariance test performed next showed insignificant $\chi^{2}$ difference value $\left(\chi^{2}(21)=20.145, \mathrm{p}=.51\right)$, suggesting that the 21 -item $\mathrm{C}$-HSQC was equivalent to both genders and it was more preferable for children in Hong Kong than the original 24-item scale.

Confirmatory factor analysis performed on the 21-item C-HSQC with combined samples showed adequate to good fit of model: $\chi^{2}(183)=363.156 ; \chi^{2} / \mathrm{df}=1.98 ; \mathrm{CFI}=.88 ; \mathrm{GFI}=.90$, $\mathrm{TLI}=.86$ and $\mathrm{RMSEA}=.06(90 \% \mathrm{CI}$ of RMSEA $=.05-.07)$. These model indicated apparent improvement as compared to the original model. Figure 2 shows the revised model. 


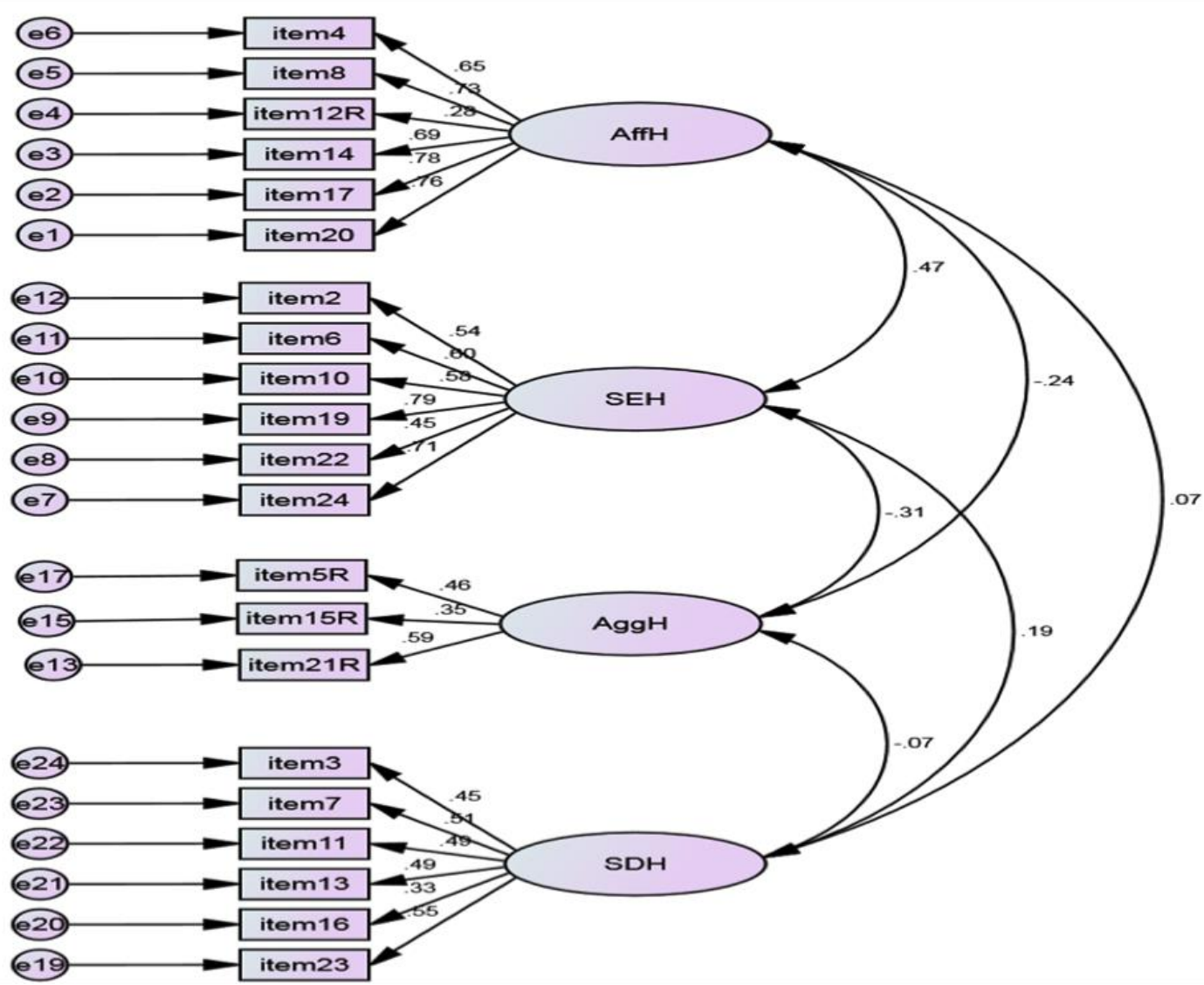

Figure 2. Confirmatory factor analysis for the four-factor model of the Chinese Humour Styles Questionnaire (C-HSQC) for Children (21 items). Note: AffH: Affiliative Humour; SEH: Self-enhancing Humour; AggH: Aggressive Humour; SDH: Self-defeating Humour.

\subsection{Concurrent and discriminant validity}

Concurrent and discriminant validity were examined by correlating the C-HSQC to the CMSHSC. A number of dimensions were positively related indicating that they all capture a certain extend of adaptive humour Table 2 showed the positive intercorrelations among the dimensions affiliative humour, self-enhancing humour, and the three dimensions of humour (C-MSHSC) ( $r$ s ranged from .36 to $.76, p s<.001)$ demonstrated that they all measure a certain degrees of adaptive humour. The highest correlations went to the relationships between affiliative humour (AffH) and humour creation (HC), and between self-enhancing humour (SEH) and humour appreciation (HA), providing basis of their concurrent validity in measuring conceptually similar functions of humour. 
Table 2. Intercorrelations between the subscales of C-HSQC and C-MSHSC

\begin{tabular}{lrrrrrrr}
\hline Variables & \multicolumn{1}{c}{ AffH } & \multicolumn{1}{c}{ SEH } & AggH & SDH & HC & HA & $\mathrm{CH}$ \\
\hline $\mathrm{AffH}^{\mathrm{a}}$ & - & & & & & & \\
$\mathrm{SEH}^{\mathrm{b}}$ & $.37^{* *}$ & - & & & & & \\
$\mathrm{AggH}^{\mathrm{c}}$ & $-.12^{*}$ & $-.22^{* *}$ & - & & & & \\
$\mathrm{SDH}^{\mathrm{d}}$ & .01 & $.13^{*}$ & -.02 & - & & & \\
$\mathrm{HC}^{\mathrm{e}}$ & $.76^{* *}$ & $.36^{* *}$ & -.07 & .04 & - & & \\
$\mathrm{HA}^{\mathrm{f}}$ & $.46^{* *}$ & $.53^{* *}$ & $-.12^{*}$ & $.12^{*}$ & $.59^{* *}$ & - & \\
$\mathrm{CH}^{\mathrm{g}}$ & $.45^{* *}$ & $.45^{* *}$ & -.09 & $.12^{*}$ & $.56^{* *}$ & $.77^{* *}$ & - \\
\hline
\end{tabular}

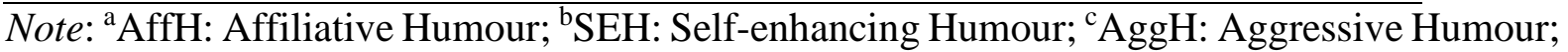
${ }^{\mathrm{d}} \mathrm{SDH}$ : Self-defeating humour; ${ }^{\mathrm{e}} \mathrm{HC}$ : Humour Creation; ${ }^{\mathrm{f}} \mathrm{HA}$ : Humour Appreciation; ${ }^{\mathrm{g}} \mathrm{CH}$ : Coping with Humour. ${ }^{*} p<.05 ; * *<.01$.

Discriminant validity was assessed by relating the two maladaptive humour styles to two adaptive styles and three dimensions of humour. For this sample, six of the correlations were found to be statistically significant. First, self-defeating humour was positively correlated with self-enhancing humour $(r=.13, p<.05)$, humour appreciation $(r=.12, p<.05)$ and coping with humour $(r=.12, p<.05)$, indicating that children who engage in self-disparaging uses of humour are more likely to enhance themselves with humour, appreciate humour, as well as using humour to cope with tough times. Second, there was a significant negative correlation between affiliative and aggressive humour $(r=-.12, p<.05)$ as well as self-enhancing and aggressive humour $(r=-.22, p<.01)$, suggesting that those who either maintain a humorous outlook on life or frequently laugh with others to facilitate interpersonal relationship are less likely to engage humour in hostile ways. These results, however, do not provide much evidence on discriminant validity of the C-HSQC for children as relationships between maladaptive humour styles with both adaptive humour styles and dimensions of humour were found.

\subsection{Convergent validity: self-esteem, coping strategies and depression}

Convergent validity was determined by examining relationships between the four humour styles with self-esteem, coping strategies, and depression (Table 3). First, adaptive and maladaptive humour styles were differentially correlated with self-esteem and depression in the predicted directions ( $r$ s ranged from -.39 to $.41, p s<.05$ to .01 ) with one exception. Specifically, there was no relationship between aggressive humour with depression, similar to the results reported by Erickson and Feldstein (2007) and Martin et al. (2003). These results suggest that C-HSQC has achieved reasonable levels of convergent validity.

Table 3. Correlations between humour styles and self-esteem, coping strategies, and depression

\begin{tabular}{lcccc}
\hline $\begin{array}{l}\text { Affiliative } \\
\text { Humour }\end{array}$ & $\begin{array}{c}\text { Self-enhancing } \\
\text { Humour }\end{array}$ & $\begin{array}{c}\text { Aggressive } \\
\text { Humour }\end{array}$ & $\begin{array}{c}\text { Self-defeating } \\
\text { Humour }\end{array}$ & $\begin{array}{c}\text { Affiliative } \\
\text { Humour }\end{array}$ \\
\hline Self-Esteem & $.35^{* *}$ & $.30^{* *}$ & $-.18^{* *}$ & $-.33^{* *}$ \\
Social Support & $.22^{* *}$ & $.32^{* *}$ & -.07 & .01 \\
Problem Solving & -.05 & $-.18^{* *}$ & .05 & -.08 \\
Escape Avoidance & $-.17^{* *}$ & -.05 & $.15^{* *}$ & $.21^{* *}$ \\
Distancing & -.05 & .07 & .01 & $.21^{* *}$ \\
Self-Control & -.06 & $.19^{* *}$ & -.09 & $-.12^{*}$ \\
CES-DC & $-.39^{* *}$ & $-.26^{* *}$ & .04 & $.41^{*}$ \\
\hline
\end{tabular}

Note: ${ }^{a}$ CES-DC - Centre for Epidemiological Studies Depression Scale. ${ }^{*} p<.05 ;{ }^{* *} p<.01$. 
With regard to coping strategies, humour styles were significantly associated with coping strategies in nine out of a possible 20 correlations. First, concerning the relationships between adaptive humour styles and coping strategies, there was a positive correlation between affiliative humour and social support $(r=.22, p<.001)$, indicating that children who joke around are more likely to look for support from others to cope. A negative correlation was also observed between affiliative humour and escape-avoidance $(r=-.17, p<.01)$, implying that children who frequently laugh with others to facilitate interpersonal relationship are less likely to employ escape-avoidance strategy in coping with difficulties. Self-enhancing humour was positively associated with social support $(r=.32, p<.01)$ and self-control $(r=.19, p<.01)$ but negatively associated with problem-solving $(r=-.18, p<.01)$, highlighting that children who maintain a humorous outlook on life are less likely to confront stressful events directly. Perhaps they are more likely to seek support from others and remain calm in dealing with stressful events.

Second, regarding the relationships between maladaptive humour styles and coping strategies, escape-avoidance was positively correlated with both aggressive and self-defeating humour $(r s=.15 \& .21, p s<.01)$, showing that children who often engage in maladaptive humour styles are more likely to avoid confronting problems. Self-defeating humour was also found to be positively correlated with distancing $(r=.21, p<.01)$, indicating children who employ humour in self-disparaging way to forget the whole thing are more likely to distance themselves from stressful events. Finally, there was a significant negative correlation between self-defeating humour and self-control $(r=-.12, p<.05)$, suggesting that children who attempt to amuse others by ingratiating themselves are less able to keep calm in emotional regulation.

These results partially support the assumptions that positive humour might be conceptualised as a form of active approach coping strategy, whereas negative humour would be associated with avoidance coping as some significant correlations were found in the predicted directions (perhaps except the significant negative correlation between selfenhancing humour and problem solving). When interpreting the strength of these relationships, cautions must be taken given some of the correlations are somewhat weaker than others.

\section{Discussion}

The purpose of this study was to translate the HSQC into Chinese and examine its psychometric properties for use among Hong Kong Chinese primary-school children.

Empirical supports were found for the factor structure of the C-HSQC. Gender equivalence of the instrument was also established as we conducted tests of configural invariance and construct-level metric invariance (Kline 2011; Millsap \& Olivera-Aguilar 2012). In sum, confirmatory factor analysis performed on the 21-item C-HSQC showed a reasonably good fit of model. Three problematic items were identified and removed, making only minimal modification to the original 24-item HSQC. However, given all three problem items belonged to the dimension of Aggressive Humour, this finding might have highlighted the possible gender and cultural differences in the uses of aggressive and self-defeating humour. One possible explanation could be that Chinese Hong Kong boys are more inclined to provide socially desirable responses in maladaptive uses of humour given a more agreeableness orientation of the Chinese culture.

Further development and revisions of items in the C-HSQC is required. A similar effort was made for the adult version of the C-HSQ (Chen, personal communiation, 2010) by removing seven items of the original 32-item HSQ that did not load well on their designated factors in the Chinese samples The resulted model with 25 items was retained for Chinese adults accordingly. 
In current findings, the high correlations between affiliative humour and humour creation, and between self-enhancing humour and coping with humour, have provided evidence for concurrent validity of the adaptive humour styles with the subscales of C-MSHSC. Concerning discriminant validity, results of the current study suggest that maladaptive humour styles were related to both adaptive humour styles and dimensions of humour. Some negative elements of humour were found in the uses of adaptive humour (e.g., children might engage in selfdefeating humour to cope and enhance themselves). However, as maintained by Martin et al. (2003: 64), it is not possible to completely disentangle the maladaptive components of humour from the more positive uses of humour. Hence, mild correlation between adaptive and maladaptive uses of humour is not unreasonable. Nevertheless, the current findings showed reasonable levels of convergent validity for the C-HSQC. As with the adult HSQ, adaptive and maladaptive humour styles were found to be differentially related to self-esteem and depression, supporting the positive and negative functions of humour and their relationships to psychological well-being. These results are generally in line with the findings of Martin et al. (2003) and Fox et al. (2013). The non-significant relationship between aggressive humour and depression supported the assertion that aggressive humour is unrelated to psychological wellbeing. To further investigate the convergent validity of the C-HSQC, the current study examined relationships between adaptive and maladaptive humour styles and coping strategies. Results suggest partial overlap between humour and coping strategies in the predicted directions. Generally, affiliative and self-enhancing humour were found to be positively correlated with social support and self-control, whilst aggressive and self-defeating humour were positively associated with escape-avoidance and distancing. These findings appear to suggest that children who score high in adaptive humour are more likely to employ positive coping strategies to deal with stressful events whilst children who score high in maladaptive humour rely more on negative coping strategies to cope, providing further evidence of the convergent validity of the questionnaire.

In sum, we translated the HSQC into Chinese and examined its psychometric properties for use among Hong Kong primary-school children. Empirical supports were found for the factor structure of the C-HSQC; with gender equivalence of the instrument was established. The C-HSQC reported reasonable convergent and divergent validity. Future research is required for further application of the scale across cultures. Since participants of the current study was drawn from only two schools, a more representative random sample could be recruited to enhance the generalisability of results. In measuring humour styles, the foremost challenge researchers must take careful note of is the limitation in capturing and reflecting individual differences when a self-report instrument is used. As Martin (2015) recently pointed out, it is certainly "important for researchers to investigate the dark side of humour in relation to well-being" (2015: 635), however, "people are usually not consciously aware of their underlying motives or the effects that their humour may be having on themselves or others" (2015: 635). As a result, it will add to the strength of any research if multiple sources of data (e.g., parents and teachers) are collected to provide alternative information and avoid potential bias in self-reported measures. Lastly, it will also be beneficial to build beyond current concept of humour by using qualitative methods to triangulate results, and to explore additional ways children engage in humour in their daily lives (Dowling 2014; Huuki et al. 2010).

\section{Appendix}

17-item Chinese version of the Multidimensional Sense of Humour Scale for Children (CMSHSC) (in English back-translation)

1. I can make other people laugh. 
2. My jokes and funny stories make others laugh.

3. I use jokes and funny stories to make my friends laugh.

4. People tell me that I say funny things.

5. I can make people laugh with the things I say.

6. I make up jokes or funny stories.

7. I like to hear a funny story.

8. I like it when people share a joke or funny story with me.

9. I like people who make me laugh.

10. I like being around people who tell jokes and funny stories.

11. I like a good joke.

12. I like people who tell jokes.

13. Jokes and funny stories help me get through tough times.

14. Using jokes and funny stories to get through tough times is a good way to go through

life.

15. I can make problems better by saying something funny.

16. Jokes and funny stories are a good way to face tough times.

17. Jokes and funny stories help to relax me.

\section{4-item Chinese version of the Humour Styles Questionnaire Children Version (C-HSQC) (in English back-translation)}

1. If someone makes a mistake, I will often tease them about it.

2. If I am feeling sad I can cheer myself up by thinking of funny things.

3. I let people laugh at me and make fun of me more than I should.

4. I find it easy to make other people laugh.

5. Even if something is really funny, I will not laugh or joke about it if it will upset someone.

6. If I have a problem I try to think of something funny about the problem to make me feel better about it.

7. I often put myself down when I am making jokes or trying to be funny.

8. I often make other people laugh by telling jokes and funny stories.

9. When I tell jokes, I'm not worried if it will upset other people.

10. If I am feeling scared I find that it helps to laugh.

11. When I am with friends or family, I seem to be the one that other people make fun of.

12. I find it hard to think of funny things to say when I'm with other people.

13. Letting others laugh at me is my way of keeping my friends and family happy.

14. I don't have to try very hard to make people laugh - I seem to be a naturally funny person.

15. I never laugh at others even if all my friends are laughing at them.

16. I often try to get other people to like me more by saying something funny about things that are wrong with me or mistakes that I make.

17. My jokes and funny stories make other people laugh.

18. Sometimes I think of something that is so funny about someone that I can't stop

myself from saying it, even if it might get me into trouble.

19. If I feel a bit uncertain in a situation it helps if I can say something funny.

20. I can make other people laugh.

21. I don't like it when people laugh at someone else to make them look silly.

22. I find that laughing and joking are good ways to cope with problems.

23. I often get carried away in putting myself down if it makes my friends or family laugh.

24. I usually try to think of something funny when I am in a difficult situation. 


\section{References}

Byrne, B. M. (2010). Structural Equation Modeling with AMOS: Basic Concepts, Applications, and Programming. New York: Routledge.

Chen, F. F., Sousa, K. H. \& West, S. G. (2005). 'Testing measurement invariance of secondorder factor models'. Structural Equation Modeling 12 (3), pp. 471-492.

Cheung, C. K., \& Yue, X. D. (2012). 'Sojourn students' humor styles as buffers to achieve resilience'. International Journal of Intercultural Relations 36 (3), pp. 353-364.

Chik, M. P. Y. (2001). Some Correlates of Children's Humor. Victoria: Monash University Doctoral Dissertation.

Dozois, D.A., Martin, R.A., \& Bieling, P.J. (2009). 'Early maladaptive schemas and adaptive/maladaptive styles of humor'. Cognitive Therapy \& Research 33 (6), pp. 585-596.

Dowling, J. S., \& Fain, J. A. (1999). 'A multidimensional sense of humor scale for school-aged children: Issues of reliability and validity'. Journal of Pediatric Nursing 14 (1), pp. 38-43.

Dowling, J. S., Hockenberry, M., \& Gregory, R. L. (2003). 'Sense of humor, childhood cancer stressors, and outcomes of psychosocial adjustment, immune function, and infection'. Journal of Pediatric Oncology Nursing 20 (6), pp. 271-292.

Dowling, J. S. (2014). 'School-age children talking about humor: Data from focus groups'. Humor 27 (1), pp. 121-139.

Erickson, S. J., \& Feldstein, S. W. (2007). 'Adolescent humor and its relationship to coping, defense strategies, psychological distress, and well-being'. Child Psychiatry \& Human Development 37 (3), pp. 255-271.

Foot, H. (1986). 'Humor and laughter'. In Hargie, O. (ed.), A Handbook of Communication Skills. Washington Square: New York University Press, pp. 355-382.

Fox, C. L., Dean, S., \& Lyford, K. (2013). 'Development of a humor styles questionnaire for children'. Humor 26 (2), pp. 295-319.

Freiheit, S. R., Overholser, J. C., \& Lehnert, K. L. (1998). 'The association between humor and depression in adolescent psychiatric inpatients and high school students'. Journal of Adolescent Research 13 (1), pp. 32-48.

Führ, M. (2002). 'Coping humor in early adolescence'. Humor 15 (3), pp. 283-304.

Gaskin, J., (2012). 'Measurement model invariance'. Gaskination's StatWiki, Available online: http://statwiki.kolobkreations.com [Accessed on 25 September 2016].

Hair, J. F., Black, W. C., Babin, B. J., \& Anderson, R. E. (2010). Multivariate Data Analysis: A Global Perspective. Boston: Pearson Education.

Hambleton, R.K. (2005). 'Issues, designs, and technical guidelines for adapting tests into multiple languages and cultures', in Hambleton, R. K., Merenda, P. F., \& Spielberg, C. D. (eds.), Adapting Educational and Psychological Tests for Cross-Cultural Assessment. Mahwah: Lawrence Erlbaum Associates Publishers, pp. 3-38.

Ho, S. K., Chik, M. P. Y., \& Chan, D. W. K. (2012). 'A psychometric evaluation on the Chinese version of the multidimensional sense of humor scale for children (C-MSHSC)'. Child Indicators Research 5, pp. 77-91.

Huck, S. W. (2012). Reading Statistics and Research. Boston: Pearson.

Huuki, T., Manninen, S., \& Sunnari, V. (2010). 'Humor as a resource and strategy for boys to gain status in the field of informal school'. Gender and Education 22 (4), pp.369-383.

Kline, R. B. (2011). Principles and Practice of Structural Equation Modeling (3rd ed.). New York: The Guilford Press.

Kuiper, N. A., \& McHale, N. (2009). 'Humor styles as mediators between self-evaluative standards and psychological well-being'. Journal of Psychology 143 (4), pp. 359-376. 
Li, H. C. W., Chung, O. K. J., \& Ho, K. Y. (2010a). 'Center for Epidemiologic Studies Depression Scale for children: Psychometric testing of the Chinese version'. Journal of Advanced Nursing 66 (11), pp. 2582-2591.

Li, H. C. W., Chung, O. K. J., Wong, L. E., \& Ho, K. Y. (2010b). 'Coping behavior checklist for Chinese children: Development and psychometric testing'. Journal of Advanced Nursing 66 (7), pp. 1633-1643.

Martin, R. A. (1998). 'Approaches to the sense of humor: A historical review', in Ruch, W. (ed.), The Sense of Humor: Explorations of a Personality Characteristic, Berlin: Mouton de Gruyter, pp. 15-60.

Martin, R. A., \& Lefcourt, H. M. (1984). 'Situational humor response questionnaire: Quantitative measure of sense of humor'. Journal of Personality and Social Psychology 47 (1), pp. 145-155.

Martin, R. A., Puhlik-Doris, P., Larsen, G., Gray, J., \& Weir, K. (2003). 'Individual differences in uses of humor and their relation to psychological well-being: Development of the Humor Styles Questionnaire'. Journal of Research in Personality 37 (1), pp. 48-75.

Martin, R. A. (2015). 'On the challenges of measuring humor styles: Response to Heintz and Ruch'. Humor 28 (4), pp. 635-639.

McGhee, P. E. (1996). The Laughter Remedy: Health, Healing and the Amuse System. Dubuque: Kendal/Hunt.

Millsap, R. E., \& Olivera-Aguilar, M. (2012). 'Investigating measurement invariance using confirmatory factor analysis', in Hoyle, R. H. (ed.), Handbook of Structural Equation modeling, New York: The Guilford Press, pp. 380-392.

Rosenberg, M. (1965). Society and the Adolescent Self-Image. Princeton: Princeton University Press.

Ruch, W. (1998). 'Sense of humor: A new look at an old concept', in Ruch, W. (ed.), The Sense of Humor: Explorations of a Personality Characteristic. Berlin: Mouton de Gruyter, pp. 314.

Sheskin, D. J. (2011). Handbook of Parametric and Nonparametric Statistical Procedures. Boca Raton: Chapman \& Hall/CRC.

Søbstad, F., \& Lillemyr, O. F. (2010). 'Humor and self-concept: A multicultural perspective'. International Research in Early Childhood Education 1, pp. 71-83.

Sugimura, N., Rudolph, K. D., \& Agoston, A. M. (2014). 'Depressive symptoms following coping with peer aggression: The moderating role of negative emotionality'. Journal of Abnormal Child Psychology 42 (4), pp. 565-575.

Svebak, S. (1996). 'The development of the Sense of Humor Questionnaire: From SHQ to SHQ6'. Humor 9 (3-4), pp. 341-361.

Taher, D., Kazarian, S. S., \& Martin, R. A. (2008). 'Validation of the Arabic Humor Styles Questionnaire in a community sample of Lebanese in Lebanon'. Journal of Cross Cultural Psychology 39 (5), pp. 552-564.

Tapley, R. (2006). 'The value of humor'. The Journal of Value Inquiry 40 (4), pp. 421-431.

Thorson, J. A., \& Powell, F. C. (1993). 'Development and validation of a multidimensional sense of humor scale'. Journal of Clinical Psychology 49 (1), pp. 13-23.

Valente, T. W. (2002). Evaluating Health Promotion Programs. New York: Oxford University Press. 